\title{
ASYMPTOTIC RELATIONS FOR PARTITIONS
}

BY

\author{
L. B. RICHMOND
}

ABSTRACT. Asymptotic relations are obtained for the number $p_{A}(n)$ of partitions of the integer $\boldsymbol{n}$ into summands from a set $A$ of integers. The set $A$ is subject to certain conditions; however the only arithmetic condition is that $A$ have property $P_{k}$ of Bateman and Erdös. A conjecture of Bateman and Erdös concerning the $k$ th differences of $p_{A}(n)$ may be verified using these asy mptotic relations.

1. Let $A=\left\{a_{0}, a_{1}, \ldots\right\}$ be an infinite set of monotonically increasing integers. Let us say, as do Bateman and Erdös [1], that $A$ has property $P_{k}$ if and only if: If we remove an arbitrary subset of $k$ elements from $A$, the remaining elements have greatest common divisor unity. If $k<0$ then any set $A$ has property $P_{k}$. In [2] we considered the number $p_{A}(n)$ of partitions of the integer $n$ into summands from $A$ when $A$ has property $P_{k}$ for all $k>0$. In this section we consider this problem when $A$ has property $P_{k}$ but not $P_{k+1}$. In the next section we investigate a conjecture of Bateman and Erdös concerning the $k$ th difference of $p_{A}(n)$. Finally in the third section we point out that a condition we assumed in [3] in order to determine the asymptotic behaviour of the moments of partition distributions is unnecessary.

For the sake of completeness we now state some definitions which are given all in [2].

Let $A(u)$ denote the number of elements of $A$ which are $\leqslant u$.

Let $f_{A}$ be defined by

$$
f_{A}(x)=\sum_{a \in A} e^{-\alpha a}
$$

Let properties (I) and (II) be defined as in [2]. It is sufficient here perhaps to state that both these properties hold when any one of (i), (ii) or (iii) below hold (see [2]).

$$
\varlimsup_{\nu \rightarrow \infty}\left(\log a_{\nu} / \log \nu\right) / \varliminf_{\nu \rightarrow \infty}\left(\log a_{\nu} / \log \nu\right)>\frac{3}{2},
$$

$$
s=\lim _{\nu \rightarrow \infty} \frac{\log \log a_{\nu}}{\log \nu} \text { exists and } s>0,
$$

Received by the editors December 6, 1974.

AMS (MOS) subject classifications (1970). Primary $10 \mathrm{~J} 20$. 


$$
A(2 u)=O\{A(u)\} \quad \text { as } u \rightarrow \infty .
$$

Let $\alpha=\alpha(n)$ be determined from

$$
n=\sum_{a \in A} a\left(e^{\alpha a}-1\right)^{-1} .
$$

Let $A_{\mu}=A_{\mu}(n)(\mu=2,3, \ldots)$ be defined as in [2] or [4]; here it will be sufficient to note that

$$
\begin{gathered}
A_{2}=\sum_{a \in A} a^{2} e^{\alpha a}\left(e^{\alpha a}-1\right)^{-2}, \\
A_{3}=\sum_{a \in A} a^{3}\left[\left(e^{\alpha a}-1\right)^{-3}+6\left(e^{\alpha a}-1\right)^{-2}+\left(e^{\alpha a}-1\right)^{-1}\right], \\
A_{4}=\sum_{a \in A} a^{4}\left[\left(e^{\alpha a}-1\right)^{-4}+36\left(e^{\alpha \alpha}-1\right)^{-3}+14\left(e^{\alpha a}-1\right)^{-2}+\left(e^{\alpha a}-1\right)^{-1}\right] .
\end{gathered}
$$

Again with $D_{\rho}=D_{\rho}(n)(\rho=1,2, \ldots)$ as defined in [2] or [4] it will be sufficient to note that $D_{1}(n)=-\sqrt{\pi / 2}\left(A_{3}^{2} / 3 A_{2}^{3}+A_{4} / 12 A_{2}^{2}\right)$.

In the following we suppose that $A$ has property $P_{k}$ but not $P_{k+1}$ for some $k \geqslant 0$. Let $\bar{A}$ denote a set of $k+1$ elements of $A$ such that $A-\bar{A}$ has g.c.d. greater than one. $\bar{A}$ is not necessarily unique; clearly the set $\{2,3\} \cup$ $\{6 i \mid i=1,2, \ldots\}$ has property $P_{0}$. We assume however that $\bar{A}$ is fixed for the remainder of this section.

Let $d$ be the g.c.d. of $A-\bar{A}$. Let $(A-\bar{A}) / d$. denote the set $\{a \mid a d \in A-\bar{A}\}$. Let $G_{A}$ be defined by

$$
G_{A}(\theta)=\prod_{a \in A} \frac{1-\exp (-\alpha a)}{1-\exp (-\alpha a+2 \pi i a \theta)} .
$$

Lemma 1.1. Let $\bar{A}, d$ and $(A-\bar{A}) / d$ be defined as above. Suppose $(A-\bar{A}) / d$ has property $P_{l}(l$ must be $\geqslant 0)$. For each fixed $\delta>0$ if

$$
\theta \in[\delta, 1-\delta]-\bigcup_{a \in \bar{A}} \bigcup_{j=1}^{a-I}\left[\frac{j}{a}-\delta, \frac{j}{a}+\delta\right] \bigcup_{j=1}^{d-1}\left[\frac{j}{d}-\delta, \frac{j}{d}+\delta\right]
$$

then $G_{A}(\theta)=O\left\{\alpha^{l+k+2}\right\}$. The O-constant depends upon $\delta$ but not $\alpha$.

Proof. From Lemma 3.4 of [2] we obtain that since $\theta$ is not within $\delta$ of any $0<j / a<1, a \in \bar{A}$,

$$
\prod_{a \in \bar{A}} \frac{1-\exp (-\alpha a)}{1-\exp (-\alpha a+i a \theta 2 \pi)}=O\left\{\alpha^{k+1}\right\}
$$

where the $O$-constant depends upon $\delta$ but not $\alpha$. Consider

$$
\bar{G}(\theta) \equiv \prod_{a \in A-\bar{A}} \frac{1-\exp (-\alpha a)}{1-\exp (-\alpha a+i a \theta 2 \pi)}
$$


for $0 \leqslant \theta \leqslant 1 / d$. Since $\bar{G}(\theta+j / d)=\bar{G}(\theta)$, this is not a restriction. Then with $\psi=d \theta$,

$$
\bar{G}_{1}(\psi) \equiv \bar{G}(\theta)=\prod_{\bar{a} \in(A-\bar{A}) / d} \frac{1-\exp (-\alpha d \bar{a})}{1-\exp (-\alpha d \bar{a}+i \bar{a} \psi 2 \pi)} .
$$

Since the set of integers $(A-\bar{A}) / d$ has property $P_{l}$, we obtain as before that for $\psi \in[\delta, 1-\delta]$

$$
\bar{G}_{1}(\psi)=O\left\{(d \alpha)^{l+1}\right\}=O\left\{\alpha^{l+1}\right\}
$$

where the $O$-constant depends upon $\delta$ but not $\alpha$.

Lemma 1.1 now follows from (1.1) and (1.2).

LemmA 1.2. Let $\epsilon>0$ be an arbitrary constant. Suppose $A$ has properties (I) and (II). Let $\bar{A}$ and $d$ be defined as above. Then there exists a constant $\delta>0$ such that

$$
\begin{aligned}
\int_{j / d-\delta}^{j / d+\delta} G_{A}(\theta) e^{2 \pi i n \theta} d \theta= & \alpha^{k+1} \prod_{a \in \bar{A}} a\left(1-e^{-i 2 \pi j a / d}\right)^{-1} \\
& \times e^{2 \pi i n j / d}\left(2 \pi A_{2}\right)^{-1 / 2}\left[1+O\left\{f_{A}^{-1 / 2+\epsilon}(\alpha)\right\}\right] .
\end{aligned}
$$

The O-constant depends upon $\delta$ but not $\alpha$.

Proof.

$$
G_{A}(j / d+\psi)=\prod_{a \in \bar{A}} \frac{1-\exp (-\alpha a)}{1-\exp (-\alpha a+2 \pi i a j / d+2 \pi i a \psi)} \cdot \bar{G}_{A}(\psi) .
$$

Now since $\bar{A}$ is a finite fixed set, we may choose $\delta$ small enough that for $|\psi| \leqslant \delta$,

$$
\prod_{a \in \bar{A}} \frac{1-\exp (-\alpha a)}{1-\exp (-\alpha a+2 \pi i a j / d)}=O\left\{\alpha^{k+1}\right\} \text {. }
$$

Also in [2] we have seen that there exists a $\delta>0$ and an $\eta$ with $0<\eta<1$ such that for $\psi_{0}=\alpha f_{A}^{-(1+\eta) / 3}(\alpha) \leqslant|\psi| \leqslant \delta$,

$$
\bar{G}_{A}(\psi)=O\left\{\alpha f_{A}^{-N}(\alpha)\right\}, \text { for each } N>0
$$

(Here we did not use the fact that $A$ had property $P_{k}$ for all $k>0$.)

For $|\psi| \leqslant \psi_{0}$ we obtain from (1.3)

Since

$$
G_{A}(j / d+\psi)=G_{A}(\psi) \cdot \prod_{a \in \bar{A}} \frac{1-\exp (-\alpha a+i 2 \pi a \psi)}{1-\exp (-\alpha a+i 2 \pi a \psi+2 \pi i a j / d)} .
$$

$$
1-e^{-\alpha a+i 2 \pi a \psi}=\alpha a+i 2 \pi a \psi+O\left\{\alpha^{2}\right\}=\alpha a[1+2 \pi i a \psi / \alpha+O\{\alpha\}]
$$
and 


$$
1-e^{-\alpha+2 \pi a \psi+2 \pi i a \psi j / d}=1-e^{2 \pi i a j / d}[1+O\{\alpha\}],
$$

we obtain that

$$
\begin{aligned}
\prod_{a \in \bar{A}} \frac{1-\exp (-\alpha a+i 2 \pi a \psi)}{1-\exp (-\alpha a+i 2 \pi a \psi+2 \pi i a j / d)}= & \alpha^{k+1} \prod_{a \in A} a\left(1-e^{-\alpha a}\right)^{-1} \\
& \times\left[\left(1+i 2 \pi \frac{\psi}{\alpha}\right)^{k+1}+O(\alpha)\right] .
\end{aligned}
$$

Thus on the range $|\psi| \leqslant \psi_{0}=\alpha f_{A}^{-(1+\eta) / 3}(\alpha)$ we obtain that

$$
\begin{aligned}
G_{A}(j / d+\psi) e^{2 \pi i n(j / d+\psi)} & \\
= & \alpha^{k+1} \prod_{a \in A} a\left(1-e^{-2 \pi i a j / d}\right)^{-1} \\
& \times e^{2 \pi i n j / d}\left[(1+2 \pi i \psi / \alpha)^{k+1}+O\{\alpha\}\right] G_{A}(\psi) e^{2 \pi i n \psi}
\end{aligned}
$$

We may expand $G_{A}(\psi) e^{2 \pi i n} \psi$ as in $[2, \S 2]$, then upon integrating from $-\psi_{0}$ to $\psi_{0}$ all odd powers of $\psi$ will drop out. Moreover

$$
\int_{-\psi_{0}}^{\psi_{0}}(\psi / \alpha)^{2} G_{A}(\psi) e^{2 \pi i n \psi} d \psi=O\left\{\alpha f_{A}^{-1}(\alpha)\right\}=O\left\{A_{2}^{-1 / 2} f_{A}^{-1 / 2+\epsilon}(\alpha)\right\}
$$

since again as in $[2, \S 2]$ we have that $A_{2}^{-1 / 2}=O\left\{\alpha f_{A}^{-1 / 2+\epsilon}(\alpha)\right\}$. Now the lemma follows from (1.3), (1.4), (1.5), and (1.6).

Lemma 1.3. Let $\epsilon>0$ be any constant. Let $A$ satisfy properties (I) and (II) of [2]. Let $\bar{A}$ be defined as above. Then there exists a constant $\delta>0$ such that for each number $j / a_{i} ; a_{i} \in \bar{A}, j=1,2, \ldots, a_{i}-1$ :

(a) if there exist $k+2$ elements $\underline{a}$ of $A$ such that $\left(a_{i} K\left(a_{i}, j\right)\right)+\underline{a}$ then

$$
\int_{j / a_{i}-\delta}^{j / a_{i}+\delta} G_{A}(\theta) e^{2 \pi i n \theta} d \theta=O\left\{\alpha^{k+2}\right\}
$$

(b) or if there exist exactly $k+1$ elements $\underline{a}$ of $A$ such that $\left(a_{i} /\left(\alpha_{i}, j\right)\right) \nmid$ $\underline{a}$, then letting $A=\{a \mid q$ defined as above $\}$, then there exists $a \delta>0$ such that

$$
\begin{aligned}
\int_{J / a_{i}-\delta}^{j / a_{i}+\delta} G_{A}(\theta) e^{2 \pi i n \theta} d \theta= & e^{2 \pi i n j / a_{i} \alpha^{k+1}} \times \prod_{a \in \bar{A}} a\left(1-e^{2 \pi i j a / a_{i}}\right)^{-1}\left(2 \pi A_{2}\right)^{-1 / 2} \\
& \times\left[1+O\left\{f_{A}^{-1 / 2+\epsilon}(\alpha)\right\}\right]
\end{aligned}
$$

Proof. The proof of part (a) is the same as the proof of Lemma 1.1. The proof of part (ii) is the same as the proof of Lemma 1.2.

THEOREM 1.1. Let $A$ have properties (I) and (II) of [2]. Suppose that A 
has property $P_{k}$ but not $P_{k+1}$ for $k \geqslant 0$. Suppose furthermore that $\overline{\lim }_{\nu \rightarrow \infty}\left[\left(\log \log a_{\nu}\right) / \log \nu\right]<\infty$. Let $m$ be any fixed integer $\geqslant 2$. Then

$$
\begin{aligned}
p_{A}(n)= & \left(2 \pi A_{2}\right)^{-1 / 2} \exp \left\{\alpha n-\sum_{a \in A} \log \left(1-e^{-\alpha a}\right)\right\} \\
& \times\left[1+\sum_{\rho=1}^{m-2} D_{\rho}+O\left\{f_{A}^{1-2 m / 3}(\alpha)\right\}+O\left\{\alpha^{k+1}\right\}\right] .
\end{aligned}
$$

Proof. As in [2]

$$
p_{A}(n)=e^{\alpha n} \int_{-1 / 2}^{1 / 2} G_{A}(\theta) e^{2 \pi i n \theta} d \theta .
$$

There is a contribution to the integral from a neighbourhood of $\theta=0$ (a saddlepoint which is determined exactly as in [2]), giving the dominant terms of Theorem 1.1. The next largest contributions come from the $\delta$-neighbourhoods $\theta \in$ $[j / d-\delta, j / d+\delta]$ and $\theta \in\left[j / a_{i}-\delta, j / a_{i}+\delta\right]$ considered in Lemmas 1.2 and 1.3 and these contributions are $O\left\{p_{A}(n) \alpha^{k+1}\right\}$. From Lemma 1.1 the integrand is $O\left\{\alpha^{k+2}\right\}$ on the remaining range of integration. However, consider the integral of $G_{A}(\theta) e^{2 \pi i n \theta}$ over the range $j / d+\delta$ to $(j+1) / d-\delta$ omitting any $\delta$-neighbourhoods of $0<k / a<1, a \in \bar{A}$. As in the proof of Lemma 1.1, this is the integral of

$$
\prod_{a \in \bar{A}} \frac{1-\exp (-\alpha a)}{1-\exp (-\alpha a+2 \pi i a d \psi)} \bar{G}_{1}(\psi) e^{2 \pi i n d \psi}
$$

from $d \delta$ to $1-d \delta$ omitting any $d \delta$-neighbourhoods of the $d k / a_{i}$ where $j / d<$ $k / a_{i}<(j+1) / d$. But $\bar{G}_{1}(\psi)=O\left\{\alpha^{2}\right\}$ except on neighbourhoods of those points considered in Lemmas 1.2 and 1.3 with $A$ replaced by $(A-\bar{A}) / d$ and from these lemmas the integral over these neighbourhoods is $O\left\{\alpha^{k+2} p_{A}(n)\right\}$. Thus the theorem follows.

REMARK. One can obtain sharper estimates from Lemmas 1.1, 1.2, and 1.3 than those given in Theorem 1.1. In general, however, the sharper estimates are rather cumbersome to state in general. Suppose $A=\{2,3\} \cup$ $\{6 i \mid i=1,2, \ldots\}$. Then one deduces that

$$
\begin{aligned}
p_{A}(n)=\frac{e^{(\pi / 3) \sqrt{n}}}{\pi^{2}}\left(\frac{3}{2}\right)^{1 / 2}\left[1+\frac{\pi}{6 n^{1 / 2}}\left(C_{0}+\right.\right. & \frac{3}{2}(-1)^{n}+\frac{2}{1-\cos (2 \pi / 3)} \\
& \times\left(\left(1-\cos \frac{2 \pi}{3}\right) \cos \frac{2 \pi n}{3}\right. \\
& \left.\left.\left.+\sin \frac{2 \pi}{3} \sin \frac{2 \pi n}{3}\right)\right)+O\left\{n^{-1}\right\}\right]
\end{aligned}
$$

where $C_{0}$ is a complicated constant which we have been unable to write in any convenient form. The oscillatory part is easy to evaluate however, and is of course independent of whether $\bar{A}$ is taken to be $\{2\}$ or $\{3\}$. 
2. In this section we shall verify, under certain asymptotic restrictions on $A$, a conjecture of Bateman and Erdös concerning $p_{A}^{(k)}(n)$ defined by

$$
\sum_{n=0}^{\infty} p_{A}^{(k)}(n) x^{n}=(1-x)^{k} \prod_{a \in A}\left(1-x^{a}\right)^{-1}
$$

We now define $\alpha, A_{\mu}$ and $D_{\rho}$ as in [5]. They are different from $\S 1$.

With Lemmas 1.1, 1.2, and 1.3 replacing Theorem 5.2 of [3] one obtains

Theorem 2.1. Let $A$ have properties (I) and (II) of [2]. Suppose that A has property $P_{s}$ but not $P_{s+1}$. Suppose furthermore that

$$
\varlimsup_{\nu \rightarrow \infty} \frac{\log \log a_{\nu}}{\log \nu}<\infty .
$$

Let $m$ be any fixed integer $\geqslant 2$ and $\epsilon$ any constant $>0$. Then for $k \geqslant 0$

$$
\begin{aligned}
p_{A}^{(k)}(n)=\left(2 \pi A_{2}\right)^{-1 / 2} \exp \left\{\alpha n-\sum_{a \in A} \log \left(1-e^{-\alpha a}\right)-k \log \left(1-e^{-\alpha a}\right)\right\} \\
\times\left[1+\sum_{p=1}^{m-2} D_{p}+O\left\{f_{A}^{1-2 m / 3}(\alpha)\right\}+O\left\{\alpha^{s+1-k}\right\}\right] .
\end{aligned}
$$

REMARK. An asymptotic result can be obtained for the case $k=s+1$ also. The result stated in complete generality is again cumbersome. Let, however, $A=\{2,3\} \cup\{6 i \mid i=1,2, \ldots\}$. In this case, one determines without too much difficulty that

$$
\begin{aligned}
p_{A}^{(1)}(n)= & \sqrt{3 / 2}\left(6 \pi n^{1 / 2}\right)^{-1} e^{(\pi / 3) \sqrt{n}} \\
\times & {\left[1+\frac{3(-1)^{n}}{2}+\frac{2}{1-\cos (2 \pi / 3)}\right.} \\
& \left.\times\left(\left(1-\cos \frac{2 \pi}{3}\right) \cos \frac{2 \pi n}{3}+\sin \frac{2 \pi}{3} \sin \frac{2 \pi n}{3}\right)+O\left\{n^{-1 / 2}\right\}\right] .
\end{aligned}
$$

More generally, one added to the contributions in Lemmas 1.2 and 1.3 gives the dominant term; hence $p_{A}^{(k+1)}(n)$ is a periodic term times $p_{A}(n) \alpha^{k+1}$ if $A$ has property $P_{k}$ but not $P_{k+1}$.

We may now say something about the conjecture of Bateman and Erdös in [5].

Let us define $\rho_{A}^{(k)}(n)=p_{A}^{(k+1)}(n) / p_{A}^{(k)}(n)$. From Theorem 2.1 and the remark following it, we obtain

THEOREM 2.2. Let $A$ have properties (I) and (II) of [2]. Suppose that $A$ 
has property $P_{\boldsymbol{k}}$. Suppose furthermore that $\overline{\lim }_{\nu \rightarrow \infty}\left[\left(\log \log a_{\nu}\right) / \log \nu\right]<\infty$. Then $\rho_{A}^{(k)}(n)=O\{\alpha\}=O\left\{n^{1 / 2}\right\}$.

PRoof. Let $\alpha_{1}$ be that in the asymptotic expression for $p_{A}^{(k+1)}(n)$ and $\alpha_{2}$ be that in the asymptotic expression for $p_{A}^{k+2}(n)$. It readily follows that $\alpha_{1}=\alpha_{2}+O\left\{\alpha_{1} f_{A}^{\epsilon-1 / 2}(\alpha)\right\}$. The second part follows from the fact that $\Sigma a\left(e^{\alpha a}-1\right)^{-1} \leqslant \Sigma_{m=1}^{\infty} m\left(e^{m \alpha}-1\right)=O\left\{\alpha^{-2}\right\}$.

REMARK. Again Theorem 2.1 and the remark following it show that under the assumptions of Theorem 2.2 one may determine the asymptotic behaviour of $\rho_{A}^{(k)}(n)$ if $A$ has property $P_{k}$. Also if $A$ does not have property $P_{k+1}$ then $\rho_{A}^{(k)}(n)$ is a periodic function of $n$ times $\alpha$ and may change sign. If $A=\{2,3\} \cup$ $\{6 i \mid i=1,2, \ldots\}$ one obtains that

$$
\begin{aligned}
\rho_{A}^{0}(n)=\frac{\pi}{6 n^{1 / 2}}[1 & +\frac{3(-1)^{n}}{2}+\frac{2}{1-\cos (2 \pi / 3)} \\
& \left.\times\left(\left(1-\cos \frac{2 \pi}{3}\right) \cos \frac{2 \pi n}{3}+\sin \frac{2 \pi}{3} \sin \frac{2 \pi n}{3}\right)+O\left\{n^{-1 / 2}\right\}\right] .
\end{aligned}
$$

3. One of the assumptions of Theorem 5.1 of [3] is that either $A$ is a $P$-sequence or that $\lim _{x \rightarrow 0} \log g_{A}(x) / \log x=0$. The proof of Theorem 1.1 shows shows that this condition is unnecessary. This condition is unnecessary in Theorem 5.2 of [3] also.

\section{REFERENCES}

1. P. T. Bateman and P. Erdös, Monotonicity of partition functions, Mathematica 3 (1956), 1-14. MR 18, 195. $389-405$.

2. L. B. Richmond, Asymptotic relations for partitions, J. Number Theory 7 (1975),

3. - The moments of partitions. II, Acta Arith. (to appear).

4. K. F. Roth and G. Szekeres, Some asymptotic formulae in the theory of partitions, Quart. J. Math. Oxford Ser. (2) 5 (1954), 244-259. MR 16, 797.

5. L. B. Richmond, Asymptotic results for partitions. II; Conjecture of Bateman and Erdös, J. Number Theory (to appear).

DEPARTMENT OF COMPUTER SCIENCE, UNIVERSITY OF MANITOBA, WINNIPEG, MANITOBA R3T 2N2, CANADA

Current address: Department of Combinatorics and Optimization, University of Waterloo, Waterloo, Ontario N2L 3G1, Canada 